\title{
根部水淹和土壤养分提升对三峡库区消落带水蓼 生长和繁殖特性的影响
}

\author{
陈禹含 ${ }^{1}$ 罗亦夫 ${ }^{1}$ 孙金星 $^{1}$ 魏冠文 ${ }^{1}$ 黄文军 $^{2}$ 罗芳丽 $^{*}$ 于飞海 $^{3}$ \\ ${ }^{1}$ 北京林业大学生态与自然保护学院, 北京 $100083 ;{ }^{2}$ 四川省林业科学研究院, 成都 $610081 ;{ }^{3}$ 台州学院湿地生态学与克隆生态学研究所/植物进化生态 \\ 学与保护浙江省重点实验室, 浙江台州 318000
}

\begin{abstract}
摘 要 水淹和土壤养分是影响三峡库区消落带植物生长的主要环境因子。消落带不同高程的植物长期经历不同的淹水强度 和土壤养分条件。该研究假设同一物种来自于消落带不同高程的植株可能产生性状分化, 从而对根部淹水和土壤养分变化具 有不同的生长和繁殖响应策略。为了验证以上假设, 选取在三峡库区消落带高低高程均广泛分布的物种水苶(Polygonum hydropiper)为研究对象, 采集自然种群的种子。在温室同质园条件下, 研究了根部水淹和土壤养分提升对高低高程水苶植株 生长和繁殖特性的影响。研究结果表明根部水淹显著或趋于显著降低了水蓼植株功能叶的叶长、叶宽、总分枝数、叶生物量、 花生物量和总生物量; 低养分处理显著或趋于显著降低了水蓼植株的总节数、总分枝数、根生物量、花生物量和总生物量, 表 明根部水淹和低土壤养分对水蓼的生长和繁殖能力具有抑制作用。同时, 根部水淹和土壤养分的交互作用显著影响植株的根 生物量, 表明根部水淹条件下高土壤养分更有利于植株根生物量的积累。高高程植株的根生物量和叶生物量显著或趋于显著 高于低高程植株, 而低高程植株的始花时间早于高高程植株, 且繁殖分配也显著高于高高程植株, 表明高低高程水蓼植株对 资源的分配策略不同。该研究结果表明水蓼的生长和繁殖特性受根部水淹和土壤养分共同限制, 但对根部水淹条件下高土壤 养分生境具有较好的适应性; 同时, 低高程植株可以通过调整其生长和繁殖特性以提高对所处生境胁迫的适应性。
\end{abstract}

关键词 三峡库区; 根部水淹; 土壤养分; 高程; 湿地植物; 繁殖分配

陈禹含, 罗亦夫, 孙釒星, 魏冠文, 黄文军, 罗芳丽, 于飞海 (2020). 根部水淹和土壤养分提升对三峡库区消落带水蓼生长和繁殖特性的影响. 植物生 态学报, 44, 1184-1194. DOI: 10.17521/cjpe.2020.0159

\section{Effects of waterlogging and increased soil nutrients on growth and reproduction of Polygonum hydropiper in the hydro-fluctuation belt of the Three Gorges Reservoir Region}

CHEN Yu-Han ${ }^{1}$, LUO Yi-Fu ${ }^{1}$, SUN Xin-Sheng ${ }^{1}$, WEI Guan-Wen ${ }^{1}$, HUANG Wen-Jun ${ }^{2}$, LUO Fang-Li ${ }^{1 *}$, and YU Fei-Hai $^{3}$

${ }^{1}$ School of Ecology and Nature Conservation, Beijing Forestry University, Beijing 100083, China; ${ }^{2}$ Sichuan Academy of Forestry, Chengdu 610081, China; and ${ }^{3}$ Institute of Wetland Ecology \& Clone Ecology/Zhejiang Provincial Key Laboratory of Plant Evolutionary Ecology and Conservation, Taizhou University, Taizhou, Zhejiang 318000, China

\footnotetext{
Abstract

Aims Flooding and soil nutrients are the main environmental factors that affect plant growth in the hydro-fluctuation belt of the Three Gorges Reservoir Region (TGRR). Flooding intensity and concentration of soil nutrients experienced by riparian plants at different elevations of the hydro-fluctuation belt are different; therefore, we hypothesized that growth and reproductive responses of plants of the same species from different elevations to waterlogging and increased soil nutrients are also different.

Methods In this study, the riparian species Polygonum hydropiper, which is widely distributed at low and high elevations of the hydro-fluctuation belt of the TGRR, was selected and its seeds were collected from natural populations. Effects of waterlogging and soil nutrients on growth and reproductive traits of $P$. hydropiper from high- and low-elevation areas were studied in a common-garden greenhouse experiment.

Important findings Waterlogging significantly, or with marginal significance, decreased length and width of functional leaves, total branch number, leaf biomass, flower biomass, and total biomass of plants; low nutrient

收稿日期Received: 2020-05-18 接受日期Accepted: 2020-09-12

基金项目：国家重点研发计划(2017YFC0505903)、国家自然科学基金(31670428)和中央高校基本科研业务费专项资金(2015ZCQ-BH-01)。Supported by the National Key R\&D Program of China (2017YFC0505903), the National Natural Science Foundation of China (31670428), and the Fundamental Research Funds for the Central Universities (2015ZCQ-BH-01).

* 通信作者Corresponding author (ecoluofangli@163.com)
} 
treatment significantly or marginal significantly decreased total node number, total branch number, root biomass, flower biomass, and total biomass of plants, indicating that both waterlogging and low soil nutrients inhibited growth and reproduction of $P$. hydropiper. Moreover, the interaction between waterlogging and soil nutrients significantly affected root biomass, showing higher root biomass accumulation at high soil nutrient conditions upon waterlogging. The high-elevation plants had significantly or marginal significantly higher leaf and root biomass than those from low elevation; however, flowering time of the low-elevation plants was significantly earlier, and reproduction allocation was higher than the high-elevation plants, indicating that resource allocation strategy was different between the high- and the low-elevation plants. The results indicate that growth and reproduction of $P$. hydropiper are inhibited by both waterlogging and soil nutrients, and this species has high adaptability at high soil nutrient conditions to waterlogging; meanwhile, low-elevation plants can adjust their growth and reproductive characteristics to improve their fitness under environmental stress.

Key words Three Gorges Reservoir Region; waterlogging; soil nutrients; elevation; wetland plant; reproduction allocation

Chen YH, Luo YF, Sun XS, Wei GW, Huang WJ, Luo FL, Yu FH (2020). Effects of waterlogging and increased soil nutrients on growth and reproduction of Polygonum hydropiper in the hydro-fluctuation belt of the Three Gorges Reservoir Region. Chinese Journal of Plant Ecology, 44, 1184-1194. DOI: 10.17521/cjpe.2020.0159

水淹是河岸带植物经常遭受的环境胁迫(Ayi et al., 2019; 郭燕等, 2019)。在水淹条件下, 光照和 溶解氧是河岸带植物生长发育的重要限制因子 (Voesenek et al., 2006; 訤杏利等, 2014)。由于水对光 的反射和散射作用(许建平等, 2014; 秦洪文等, 2017), 水淹会显著降低水下的光照强度(秦洪文等, 2017); 同时, 氧气在水中的溶解度和扩散速率均非 常低, 其溶解度和扩散系数分别为: $1.22 \times$ $10^{-3} \mathrm{~mol} \cdot \mathrm{L}^{-1}$ (标准大气压下 $25{ }^{\circ} \mathrm{C}$ 纯水中)和 $2.1 \times$ $10^{-5} \mathrm{~cm}^{2} \cdot \mathrm{s}^{-1}$ (仅约为空气中扩散系数的 $1 / 10000$ )(刘 泽涁等, 2013; Xing et al., 2014; 许建平等, 2014; 杜 珲等, 2016)。低的水下光照强度和氧气浓度会抑制 植物的光合能力, 降低其光合速率、气孔导度、蒸 腾速率以及光合色素的含量(刘泽涁等, 2013; 李晓 雪等, 2019; 章毅等, 2019)。此外, 水淹还会通过影 响消落带不同高程土壤的理化性质，间接影响河岸 带植物的生长发育。周期性的淹水和退水会导致土 壤养分流失(朱强等, 2014), 同时, 淹水也可能带来 泥沙沉积而提高土壤养分含量(王娅儆等, 2016a, 2016b; 程瑞梅等, 2017)。有研究发现消落带高低高 程的土壤养分含量存在较大差异, 土壤的全氮、全 磷和全钾含量随高程的增加, 呈先增加后降低的趋 势(王娅儆等, 2016a, 2016b)。

应对周期性的自然水淹, 河岸带植物表现出形 态性状上的适应性, 比如通气组织的形成、叶片变 薄、茎伸长生长以及气生根的生成等(Phukan et al., 2016; Wang et al., 2016; Heydel et al., 2017; Hua et al., 2017); 同时, 河岸带植物的繁殖特性也随着
淹水而发生变化, 水淹耐受能力较强的物种往往能 有效地利用退水或两次淹水中间的间隙快速地完成 其繁殖过程; 这些植物可以通过调整繁殖期、缩短 花期以降低或避免淹水对繁殖过程的影响，例如稗 (Echinochloa crus-galli)的种子可在水位下降后迅速 萌发以提高其子代的存活率(Colmer \& Voesenek, 2009; 陶敏等, 2011; 樊大勇等, 2015; Su et al., 2016)。河岸带不同高程的植物所经历的水淹胁迫往 往是不同的, 分布在高高程的植物经历的淹水频率 较低, 时长更短; 而分布在低高程的植物经历的淹 水频率更高且时长更长(Chen \& Xie, 2007; 袁慎鸿 等, 2014)。已有研究表明不同高程的植物群落特征 如物种数和优势种存在较大差异, 物种数随高程的 升高表现出增加的趋势(张爱英等, 2018)。由于长期 遭受不同的淹水强度, 河岸带不同高程植物所受 到环境选择压力也是不同的; 因此，同一物种分 布在不同高程的植株的生长和繁殖特性也可能存 在差异。

三峡工程是世界上最大的水利水电枢纽工程 (Wu et al., 2004)。三峡水库夏季水位降至145 m, 冬 季蓄水至 $175 \mathrm{~m}$, 形成了与自然河流相反的“冬蓄夏 排”周期性水位变化节律(王业春等, 2012; 赵琴和 陈教斌, 2018)。由于大坝的截流作用, 水库内水流 速度非常缓慢, 接近于静止水体(Su et al., 2017)。水 位周期性变化形成大面积的消落带区域，其高低高 程水位落差最大可达 $30 \mathrm{~m}$ (雷波等, 2014; 郭燕等, 2019)。三峡库区高高程区域的植物经历的水淹时间 较短且深度较浅, 而低高程区域的植物经历的水淹 
时间较长且深度较深(刘维暐等, 2012; Chen et al., 2019b)。除了三峡库区以外, 国内外河流自然消落 区的高程落差很少能达到 $10 \mathrm{~m}$ 以上，通过控制实验 就更难实现较大的高程差(Schaff et al., 2003; Park \& Kim, 2020)。因此, 目前针对高程对河岸带植物生 长和繁殖特性影响的研究仍较为缺乏。仅有少数 学者对高程的效应进行了初步的探讨, 但其研究 样地海拔落差仍不超过5 m (Chen et al., 2019a, 2019b)。

水蓼(Polygonum hydropiper) 是蓼科苶属(Polygonum)的一年生草本植物, 自然生长在河(湖)岸边, 在我国分布较为广泛(李安仁, 1998)。同时, 该物种 也具有非常发达的地下根状茎, 节处可着生细根 和幼芽(Chen et al., 2019b)。植株可以以地下根状茎 越冬, 翌年春季萌发形成新的分株。水蓼对湿地自 然水位变化具有较强的适应能力, 能适应陆生和 水生生境, 是很多湿地的优势植物群落类型(Chen et al., 2020)。已有研究表明水苶对水淹具有一定的 适应性(陈芳清等, 2008; Chen \& Xie, 2011)。在淹水 条件下, 植株可以通过改变叶片形状、形成不定根 以及改变生物量分配等方式来适应淹水环境( Pan et al., 2014)。该物种是三峡库区消落带的常见物种, 在消落带的不同高程具有大面积的自然分布(Chen et al., 2020)。

河(湖)岸带的土壤水分和养分含量往往沿垂直 分布高度的不同而存在差异, 湿地或两栖植物如

Ranunculus reptans和Solanum dulcamara分布在不 同区域的植株通过种内分化, 表现出不同的形态适 应特征以较好地适应所在生境(Lenssen et al., 2004; Zhang et al., 2016); 同时, 也有研究发现某些物种 的种内分化即使在较小空间尺度下也能发生 (Lenssen et al., 2004)。由于分布在消落带不同高程 的水蓼植株长期经历不同的淹水强度和土壤养分环 境, 因此, 我们假设来自不同高程的水蓼植株可能 产生了性状分化, 从而形成不同的生长和繁殖策略 以适应不同的淹水和土壤养分条件。为了验证以上 假设, 本研究以三峡库区消落带高低高程区域的水 苶为研究对象, 在温室同质园条件下, 研究了不同 的淹水和土壤养分处理对来源于高低高程水苶植株 的形态、生物量和繁殖性状的影响, 以期能明确不 同高程水苶植株对根部水淹和土壤养分提升的生长 和繁殖响应, 为消落带植被的保护与恢复提供一定
的理论依据。

\section{1 材料和方法}

\section{1 实验材料与地点}

在三峡库区消落带重庆北碚至湖北宜昌区域选 取了 3 个样地, 在每个样地各选取高低高程的 2 个水 苶自然种群, 分别为: 重庆奉节县草堂镇梨木咀的 高高程种群 $\left(173 \mathrm{~m} ; 31.07^{\circ} \mathrm{N}, 109.60^{\circ} \mathrm{E}\right)$ 和低高程种 群 $\left(153 \mathrm{~m} ; 31.07^{\circ} \mathrm{N}, 109.60^{\circ} \mathrm{E}\right)$, 重庆巴东县渡河镇 老屋场的高高程种群 $\left(172 \mathrm{~m} ; 31.20^{\circ} \mathrm{N}, 110.30^{\circ} \mathrm{E}\right)$ 和 低高程种群 $\left(156 \mathrm{~m} ; 31.20^{\circ} \mathrm{N}, 110.30^{\circ} \mathrm{E}\right)$, 湖北剓归 县归州镇官庄坪村乔家坝的高高程种群 (166 m; $\left.31.00^{\circ} \mathrm{N}, 110.76^{\circ} \mathrm{E}\right)$ 和低高程种群 $\left(155 \mathrm{~m} ; 31.00^{\circ} \mathrm{N}\right.$, $\left.110.76^{\circ} \mathrm{E}\right)$ 。在每个种群随机采集9株成熟水苶植株 的种子, 相邻植株间距至少 $2 \mathrm{~m}$ 。种子自然风干并混 合后, 用于温室实验。

\section{2 种子垂直方向上的潜在扩散距离计算}

为了探究水蓼种子是否会随水流在高低高程间 扩散, 我们采用以下公式计算了水蓼种子在垂直方 向上的潜在扩散距离: 种子垂直方向上的潜在扩散 距离=种子最长漂浮时间 $\times$ 水位上涨速度。种子最长 漂浮时间: 每个种群随机挑选 100 粒成熟饱满的风 干种子, 高低高程各 300 粒, 作为 3 个重复。将种子分 别放入 6 个装有 $400 \mathrm{~mL}$ 自来水的烧杯(体积: $500 \mathrm{~mL}$ ) 中, 轻微搅动水体, 记录所有种子沉底的时间。水位 上涨速率根据2009-2018 年全国水雨情信息网 (http://xxfb.mwr.cn/sq_djdh.html)每日水位数据计算 得出, 即每年水库蓄水期间水位上涨最快的 3 天数 据的平均值。

\section{3 实验设计}

2018年6月, 每个种群选择500粒成熟饱满的种 子, 放在河沙为基质的培养皿中进行萌发。每个种 群随机选取 24 株大小高度一致的实生苗移栽于塑料 花盆(盆口直径 $13.1 \mathrm{~cm}$, 盆底直径 $9.2 \mathrm{~cm}$, 盆高 $11.3 \mathrm{~cm}$ )中, 共 144 株植株, 具体实验设计为: 6 种群 $\times$ 2 个淹水处理 $\times 2$ 个土壤养分处理 $\times 6$ 重复。淹水处理 包括不水淹(正常浇水)和根部水淹处理(从花盆底部 向上淹水 $5 \mathrm{~cm})$ 。土壤养分处理包括高养分和低养分 处理, 两者的草炭:沙:壤土的体积比分别为: $1: 1: 3$ 和 1:3:1; 养分含量分别为: $\mathrm{N},(1.51 \pm 0.03) \mathrm{mg} \cdot \mathrm{g}^{-1}, \mathrm{P}$, $(1.46 \pm 0.03) \mathrm{mg} \cdot \mathrm{g}^{-1} ; \mathrm{N},(0.48 \pm 0.03) \mathrm{mg} \cdot \mathrm{g}^{-1}, \mathrm{P},(0.55 \pm$ $0.04) \mathrm{mg} \cdot \mathrm{g}^{-1}$ 。土壤养分梯度设置依据我们前期对三 
峡库区消落带高低高程土壤养分含量的测定结果, 以 及近期发表的相关文献中该区域的土壤养分含量的 变化范围: $\mathrm{N},(0.40-1.92) \mathrm{mg} \cdot \mathrm{g}^{-1} ; \mathrm{P},(0.43-2.47) \mathrm{mg} \cdot \mathrm{g}^{-1}$ (Ye et al., 2019; Wang et al., 2020; Wei et al., 2020)进 行设定。实验采用裂区实验设计, 高养分和低养分 处理的 72 株植株分别放置于 12 个塑料箱中 $($ 长 $41 \mathrm{~cm}$, 宽 $31 \mathrm{~cm}$, 高 $14.5 \mathrm{~cm})$, 每个塑料箱中放置6株来自 不同样地不同高程的植株。从高养分和低养分处理 中随机选择 6 个塑料箱, 对其中的植株进行根部水 淹处理, 另外 6 个塑料箱中的植株无水淹处理。当大 部分植株进入繁殖期后, 对植株进行收获。实验时 间共154天。实验在北京林业大学园林实验教学示范 中心开展。

\section{4 生长和繁殖指标测定}

在收获植物之前, 测定每株植株的主茎长、总 节数(主茎节数+分枝节数)、总分枝数、功能叶叶长 和叶宽, 以及始花时间(种子开始萌发后到开始开 花的时间)。然后, 对每株植株的根、茎、叶和花分 别进行收获, 放入 $70{ }^{\circ} \mathrm{C}$ 烘箱中烘至恒质量, 获取植 株各部分的生物量。通过计算花生物量与总生物量 的比值获得植株的繁殖分配。

\section{5 数据分析}

在数据分析之前, 对数据的方差齐性和正态性 进行了检验。为了提高数据的正态性, 将叶长和茎 生物量的数据转化为 $\lg (x+1)$ 值, 根生物量的数据 转化为平方根。使用三因素方差分析检验根部水淹、 土壤养分和高程及其交互作用对水蓼植株的形态 (功能叶叶长、叶宽, 主茎长, 主茎节数, 总节数和 总分枝数), 生物量(根、茎、叶和总生物量)和繁殖 (始花时间、花生物量和繁殖分配) 性状的影响。数 据分析在SPSS 22.0中完成, 并使用Origin Pro 2017 作图。

\section{2 结果}

\section{1 种子垂直方向上的潜在扩散距离}

通过种子漂浮实验得出, 高低高程水蓼风干 种子最长漂浮时间分别为(11.1 \pm 0.2$)$ 和(10.4 \pm 0.2$)$ $\mathrm{h}$ 。2009至2018年水库蓄水期间高流量引起的水位 上涨速率为 $1.1-3.2 \mathrm{~m} \cdot \mathrm{d}^{-1}$ (附录 $\mathrm{I}$ )。根据计算高低高 程水蓼种子垂直方向上的潜在扩散距离分别为 $(0.9$ $\pm 0.1)$ 和 $(0.8 \pm 0.1) \mathrm{m}$, 因此, 高低高程种群的种子 可能随水流传播的距离较短。由于种子采集样地高
低高程种群间的高程差为11-20 m, 因此, 低高程 种群的种子不可能随着水位上涨扩散至高高程区 域; 此外, 根据采样时的观察, 我们发现部分高高 程植株是通过地下根状茎越冬后产生的分株, 因此, 高高程的植株来源于高高程种群的种子或地下根 状茎。

\section{2 根部水淹和土壤养分提升对高低高程水蓼植 株形态性状的影响}

根部水淹显著影响水蓼植株功能叶的叶长和叶 宽, 趋于显著影响植株的总分枝数(表1)。根部水淹 植株的功能叶长、叶宽和总分枝数均低于无水淹处 理植株(图1 A、1B、1F)。土壤养分趋于显著影响植 株的总节数和总分枝数(表1), 高养分处理植株的总 节数和总分枝数高于低养分处理植株(图1E、1F)。 根部水淹、土壤养分和高程的交互作用对水苶形态 指标的影响不显著(表1)。

\section{3 根部水淹和土壤养分提升对高低高程水苶植 株生物量性状的影响}

根部水淹显著影响水蓼植株的总生物量, 趋于 显著影响植株的叶生物量(表1)。根部水淹植株的总 生物量和叶生物量低于无水淹处理植株(图2C、 2D)。土壤养分显著影响植株的根生物量, 趋于显著 影响植株的总生物量(表1)。高养分处理植株的总生 物量和根生物量高于低养分处理植株(图2A、2D)。 高程显著影响植株的叶生物量, 趋于显著影响植株 的根生物量(表1)。高高程植株叶生物量和根生物量 高于低高程植株(图2A、2C)。根部水淹和土壤养分 的交互作用显著影响植株的根生物量, 无水淹处理 下高低养分处理植株的根生物量无显著差异, 而根 部水淹处理下高养分处理植株的根生物量显著高于 低养分处理植株(表1; 图2A)。

\section{4 根部水淹和土壤养分提升对高低高程水苶植 株繁殖性状的影响}

根部水淹显著影响水蓼植株的花生物量, 根部 水淹植株的花生物量显著低于无水淹处理的植株 (表1; 图3B)。土壤养分趋于显著影响植株的花生物 量, 高养分处理植株的花生物量高于低养分处理植 株(表1; 图3B)。高程显著影响植株的始花时间和繁 殖分配(表1)。低高程植株的始花时间显著早于高高 程植株(图3A); 同时, 其繁殖分配也显著高于高高 程植株(图3C)。根部水淹、养分和高程的交互作用 对植株繁殖性状无显著影响(表1)。 
表1 根部水淹、土壤养分和高程对水蓼形态、生物量和繁殖性状的影响

Table 1 Effects of waterlogging, soil nutrients, and elevation on morphological, biomass, and reproductive traits of Polygonum hydropiper

\begin{tabular}{|c|c|c|c|c|c|c|c|c|}
\hline 性状 Trait & 根部水淹 & Waterlogging $(\mathrm{W})$ & 养分 Nutrient (N) & 高程 Elevation (E) & $\mathrm{E} \times \mathrm{W}$ & $\mathrm{E} \times \mathrm{N}$ & $\mathrm{W} \times \mathrm{N}$ & $\mathrm{W} \times \mathrm{N} \times \mathrm{E}$ \\
\hline \multicolumn{9}{|l|}{ 形态性状 Morphological trait } \\
\hline 功能叶长 Length of functional leaf & & $7.91^{* *}$ & 0.99 & 0.45 & 0.31 & 0.01 & 0.11 & 0.01 \\
\hline 功能叶宽 Width of functional leaf & & $3.97^{*}$ & 0.01 & 0.80 & 0.02 & 0.07 & 0.21 & 0.00 \\
\hline 主茎长 Main stem length & & 0.07 & 1.45 & 1.98 & 0.39 & 0.38 & 0.16 & 0.05 \\
\hline 主茎节数 Node number of main stem & & 0.36 & 2.41 & 0.17 & 0.28 & 2.22 & 1.09 & 0.00 \\
\hline 总节数 Total node number & & 2.74 & $3.04^{\#}$ & 1.14 & 2.17 & 1.45 & 0.64 & 0.01 \\
\hline 总分枝数 Total branch number & & $2.97^{\#}$ & $3.87^{\#}$ & 0.55 & 2.18 & 0.55 & 0.55 & 0.06 \\
\hline \multicolumn{9}{|l|}{ 生物量性状 Biomass trait } \\
\hline 根生物量 Root biomass & & 2.58 & $5.08^{*}$ & $3.03^{\#}$ & 0.84 & 0.20 & $4.28^{*}$ & 0.71 \\
\hline 茎生物量 Stem biomass & & 1.88 & 0.86 & 1.27 & 2.71 & 0.34 & 2.26 & 0.05 \\
\hline 叶生物量 Leaf biomass & & $3.55^{\#}$ & 0.74 & $5.44^{*}$ & 0.72 & 0.19 & 2.70 & 0.00 \\
\hline 总生物量 Total biomass & & $6.74^{*}$ & $2.87^{\#}$ & 0.46 & 2.43 & 0.52 & 1.20 & 0.14 \\
\hline \multicolumn{9}{|l|}{ 繁殖性状 Reproductive trait } \\
\hline 始花时间 First flowering time & & 0.37 & 0.09 & $4.05^{*}$ & 0.00 & 0.58 & 0.33 & 0.17 \\
\hline 花生物量 Flower biomass & & $10.44^{* *}$ & $2.98^{\#}$ & 2.59 & 0.14 & 1.57 & 1.47 & 0.16 \\
\hline 繁殖分配 Reproduction allocation & & 2.36 & 0.44 & $6.75^{*}$ & 0.50 & 0.23 & 2.48 & 0.30 \\
\hline
\end{tabular}

表中数据为 $F$ 值。方差检验的显著性水平: ***, $p<0.001 ; * *, p<0.01 ; *, p<0.05 ; \#, 0.05 \leqslant p<0.1$; 无标注即为 $p \geqslant 0.1$ 。当 $p<0.05$ 时, $F$ 值加粗显示。 始花时间、花生物量和繁殖分配的自由度为 $(1,133)$, 其余指标的自由度均为 $(1,135)$ 。

Values are $F$ and symbols show $p(* * *, p<0.001 ; *, p<0.01 ; *, p<0.05 ; \#, 0.05 \leqslant p<0.1$, and no symbols $p \geqslant 0.1)$. Values of $p<0.05$ are in bold. Degree of freedom is $(1,133)$ for first flowering time, flower biomass and reproduction allocation, and degree of freedom is $(1,135)$ for other indexes.
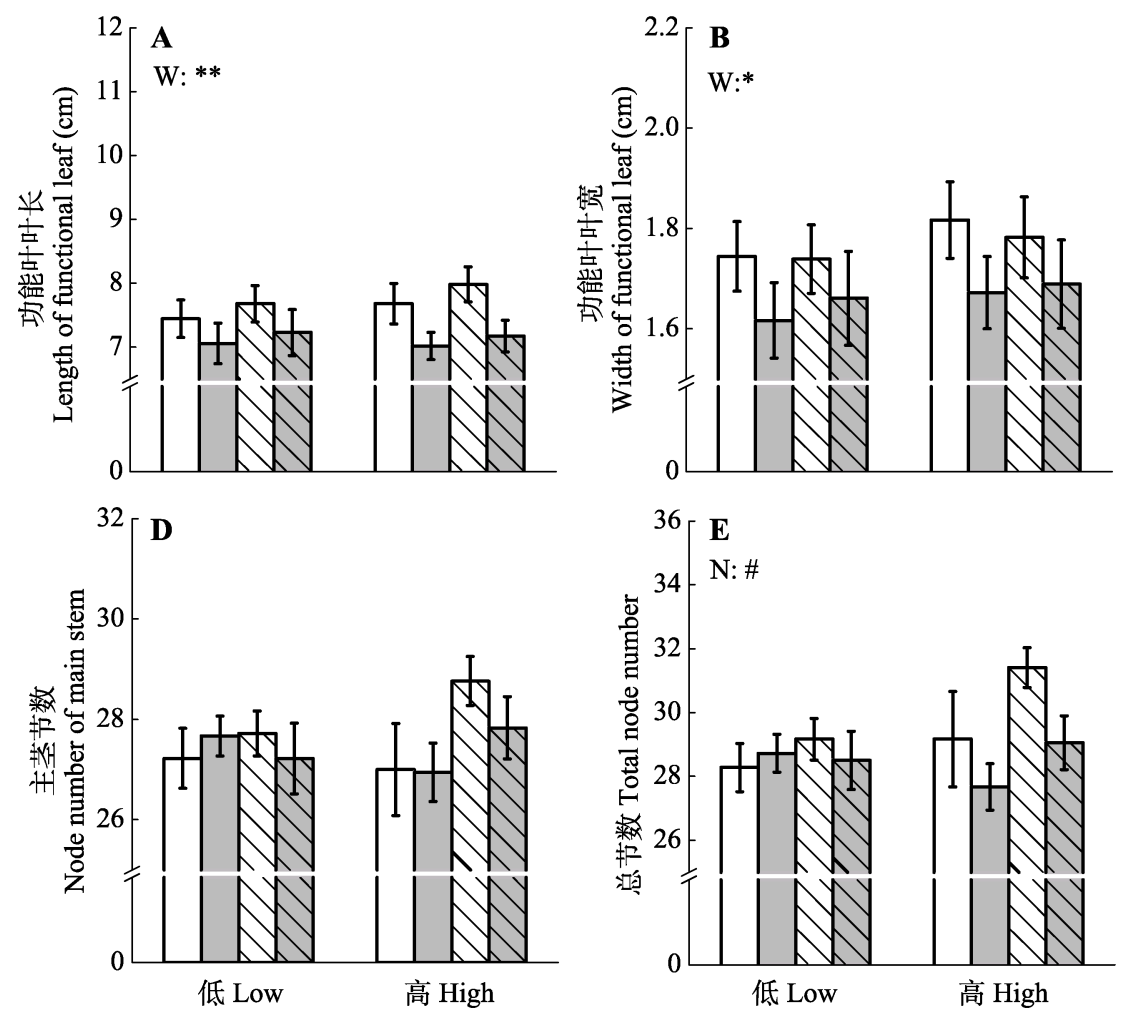
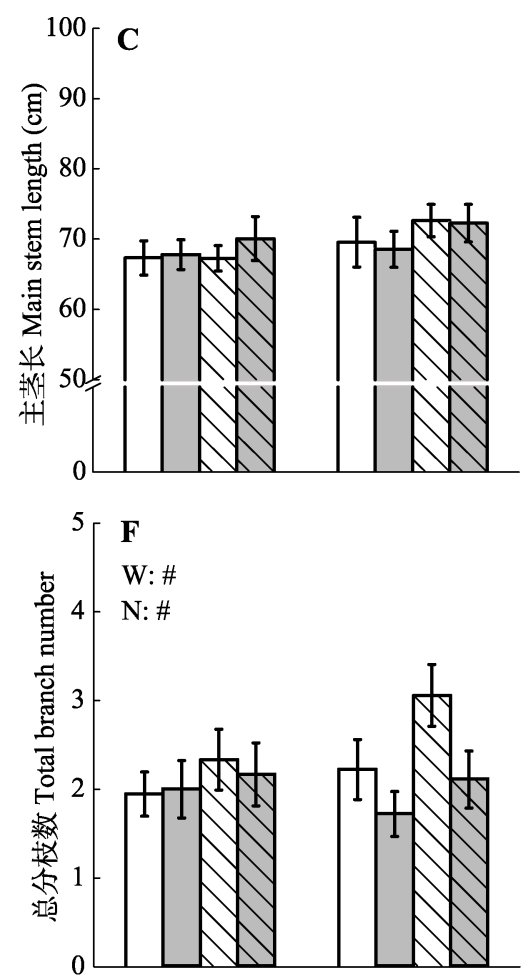

低 Low

高程 Elevation

低养分+不水淹 Low nutrient without waterlogging $\triangle \nabla$ 高养分+不水淹 High nutrient without waterlogging

低养分+根部水淹 Low nutrient with waterlogging $\triangle \nabla$ 高养分+根部水淹 High nutrient with waterlogging

图1 不同根部水淹 $(\mathrm{W})$ 和土壤养分 $(\mathrm{N})$ 处理下高低高程水蓼植株的功能叶叶长 $(\mathbf{A})$ 、叶宽 $(\mathbf{B})$ 、主茎长 $(\mathbf{C})$ 、主茎节数 $(\mathbf{D})$ 、总节数 $(\mathbf{E})$ 和总分枝数 $(\mathbf{F})$ (平均值土标准误差)。 ${ }^{* *}, p<0.01 ; *, p<0.05 ; \#, 0.05 \leqslant p<0.1$ 。

Fig. 1 Length (A) and width (B) of functional leaf, main stem length (C), node number of main stem (D), total node number (E), and total branch number (F) of Polygonum hydropiper from high and low elevations under different waterlogging (W) and soil nutrients $(\mathrm{N})$ treatments (mean $\pm S E$ ). ${ }^{* *}, p<0.01 ;{ }^{*}, p<0.05 ; \#, 0.05 \leqslant p<0.1$.

www.plant-ecology.com 

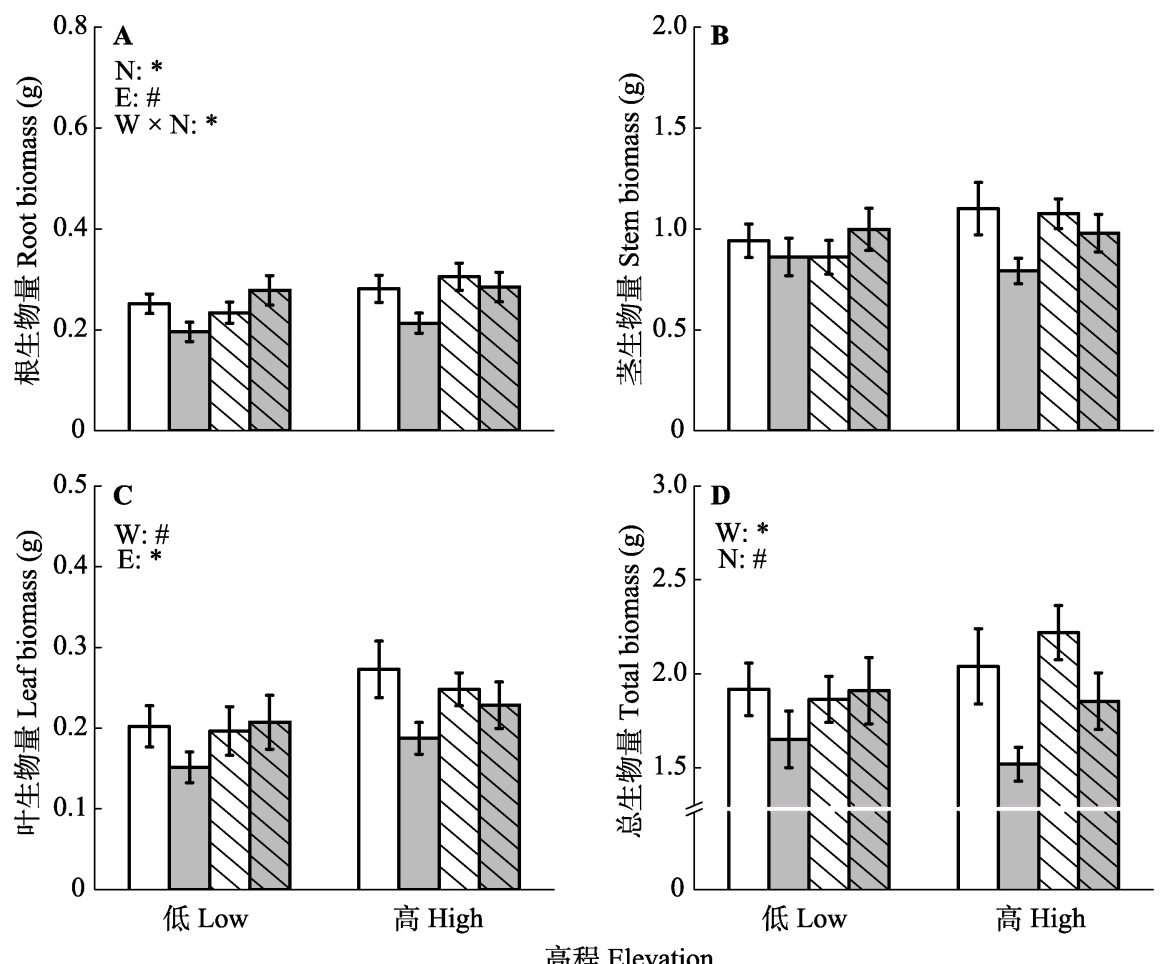

低养分+不水淹 Low nutrient without waterlogging $\triangle \backslash$ 高养分+不水淹 High nutrient without waterlogging 低养分+根部水淹 Low nutrient with waterlogging $\triangle \backslash$ 高养分+根部水淹 High nutrient with waterlogging

图2 不同根部水淹 $(\mathrm{W})$ 和土壤养分 $(\mathrm{N})$ 处理下高低高程 $(\mathrm{E})$ 水蓼植株的根生物量 $(\mathbf{A})$ 、茎生物量 $(\mathbf{B})$ 、叶生物量 $(\mathbf{C})$ 和总生物量 (D)(平均值土标准误差)。 $*, p<0.05 ; \#, 0.05 \leqslant p<0.1$ 。

Fig. 2 Root biomass (A), stem biomass (B), leaf biomass (C), and total biomass (D) of Polygonum hydropiper from high and low elevations (E) under different waterlogging $(\mathrm{W})$ and soil nutrients $(\mathrm{N})$ treatments (mean $\pm S E$ ). ${ }^{*}, p<0.05 ; \#, 0.05 \leqslant p<0.1$.
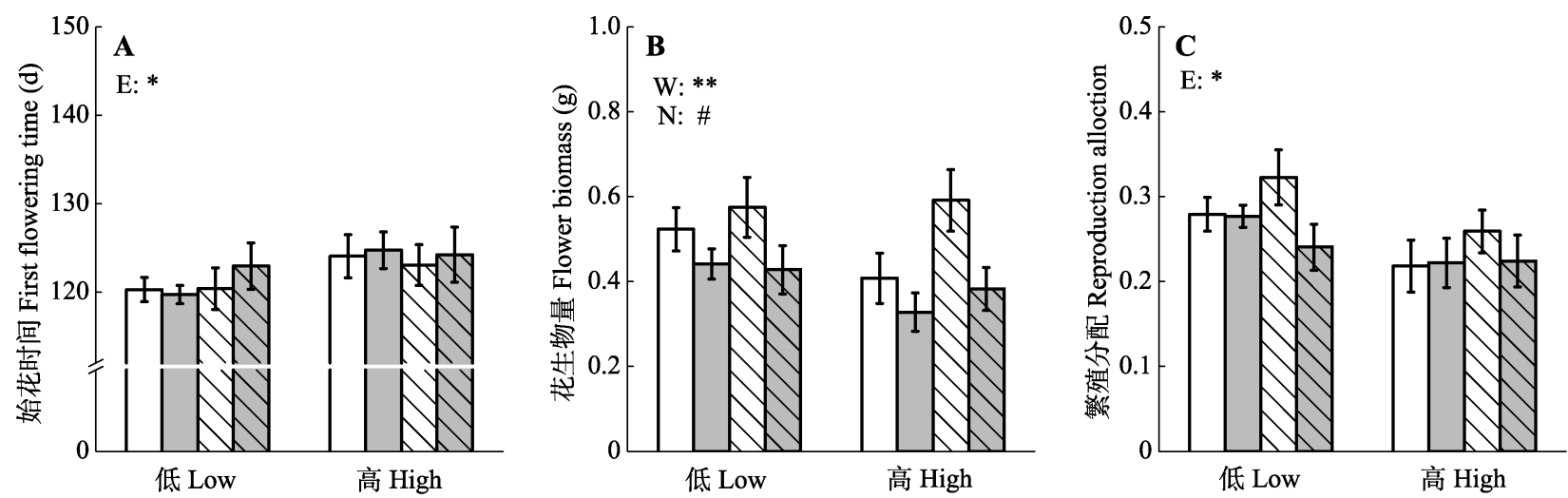

高程 Elevation

低养分+不水淹 Low nutrient without waterlogging

低养分+根部水淹 Low nutrient with waterlogging

$\triangle \searrow$ 高养分+不水淹 High nutrient without waterlogging

$\triangle \searrow$ 高养分+根部水淹 High nutrient with waterlogging

图3 不同根部水淹 $(\mathrm{W})$ 和土壤养分 $(\mathrm{N})$ 处理下高低高程 $(\mathrm{E})$ 水蓼植株的始花时间 $(\mathbf{A})$ 、花生物量 $(\mathbf{B})$ 和繁殖分配 $(\mathbf{C})($ 平均值土标准 误差)。**, $p<0.01 ;^{*}, p<0.05 ; \#, 0.05 \leqslant p<0.1$ 。

Fig. 3 First flowering time (A), flower biomass (B), and reproduction allocation (C) of Polygonum hydropiper from high and low elevation (E) under different waterlogging (W) and soil nutrients $(\mathrm{N})$ treatments (mean $\pm S E$ ). ${ }^{* *}, p<0.01 ; *, p<0.05 ; \#, 0.05 \leqslant$ $p<0.1$.

\section{3 讨论}

在三峡库区消落带区域, 淹水和土壤养分往往
共同影响河岸带植物的生长和发育(张志永等, 2016; Zhou et al., 2020)。本研究结果表明根部水淹抑制了 水苶植株的叶生长和分株的形成, 降低了其花生物 
量和总生物量(表1; 图1-3)。这与已有的研究结果一 致——淹水显著降低了翮草(Phalaris arundinacea)、 牛鞭草 (Hemarthria altissima)、狗牙根 (Cynodon dactylon)等植物的生物量积累(李秋华等, 2013)。这 可能与植物在水淹条件下光合能力受到抑制有关 (刘泽涁等, 2013; 李晓雪等, 2019; 章毅等, 2019); 同时, 也有研究发现植物在水淹条件下可通过落叶 以降低其呼吸消耗，提高其对水淹的适应性(Striker et al., 2011; Ye et al., 2018)。

低土壤养分处理显著抑制了水蓼植株根的生长, 在无水淹处理下该效应不显著, 而在根部水淹条件 下高养分处理植株的根生物量显著高于低养分处理 植株(表 1 ; 图2; 根部水淹 $\times 土$ 壤养分效应显著); 研 究结果表明在根部水淹条件下水蓼植株对土壤养分 更为敏感, 高的土壤养分有利于提高植株根生物量 的积累。本研究的根部水淹处理中, 植物根系并未 完全被水淹没, 部分根系仍在水面以上(土壤表面 往下大约 $4 \mathrm{~cm}$ ), 缺氧环境可能对根系养分吸收能 力的影响不大。其次, 根部水淹条件下低高程植株 对高养分环境具有较强的适应性, 这可能是由于高 低高程的水蓼植株在长期适应不同水淹环境的过程 中形成了种内分化, 从而表现出不同植株的性状差 异, 这种现象在一些杨柳科和禾本科植物适应昆虫 采食、土壤养分、水分胁迫中也得到了验证(Soininen et al., 2013; Abbott \& Stachowicz, 2016; Barker et al., 2019)。

高高程水苶植株的叶和根生物量显著或趋于显 著高于低高程植株(图2), 表明高高程植株将更多的 生物量投入到营养生长。根据三峡水库的水位调度 方案, 与低高程植株相比, 每年消落带高高程区域 被水淹的时间较晚而退水的时间较早 ( $\mathrm{Su}$ et al., 2012; Fan et al., 2015; 樊大勇等, 2015; 由永飞等, 2017)。因此, 高高程植株水淹间歇的恢复时间比低 高程植株更长; 植株将更多的生物量分配到叶和根 的生长, 有利于植株光合产物的积累和对土壤养分 的吸收(Yan et al., 2015; 徐金英等, 2016; Chen et al., 2019b)。已有的研究也发现在相对较浅的水淹环境 下河岸带植物如狗牙根、三江蔍草 (Scirpus nipponicus)和桐花树(Aegiceras corniculatum)等具有 较高的叶和根生物量分配(洪明等, 2011; 罗美娟等, 2012; 古勇波等, 2019)。

低高程水蓼植株的始花时间显著早于高高程植
株，且繁殖分配显著高于高高程植株(表1; 图3)，表 明低高程植株将更多的生物量投入到生殖生长。由 于低高程植株经历的淹水频率更高、历时更长, 并 且已有研究表明该物种并不能耐受长时间的完全水 淹(陈芳清等, 2008; Chen \& Xie, 2011), 因此, 该物 种在淹水强度高的区域可能通过改变其始花时间并 增加对其有性繁殖的投入, 以提高子代对胁迫环境 的适应性(陈芳清等, 2008; Chen \& Xie, 2011; Chen et al., 2014)。已有研究表明河岸带植物可以通过改 变其繁殖策略以提高对水淹胁迫的适应性, 比如典 型河岸带植物秋华柳(Salix variegata)可以通过推迟 其始花时间和结实期、快速完成繁殖过程以及改变 繁殖分配等策略以避开水淹周期(苏晓否等, 2010; Nam et al., 2017; 何莣廷等, 2020)。

在胁迫环境下, 植物有限的物质积累决定了它 们需要在繁殖、生长和生存之间进行权衡, 即营养 生长和生殖生长之间的资源竞争(李亚芳等, 2016); 同时, 也有研究表明胁迫环境下植物会优先将资源 分配至生殖生长(Villellas \& Garcia, 2018), 比如毛 茛科的一年生植物在淹水时长增加的情况下会提高 对繁殖器官的投资(Mony et al., 2010)。由于植物的 繁殖特性受环境影响较为显著, 低高程淹水环境对 水蓼母代植株繁殖特性的影响也可能影响其子代的 繁殖策略(苏晓否等, 2010; 李亚芳等, 2016)。

\section{4 结论}

根部水淹和土壤养分提升对河岸带植物水苶的 生长和繁殖特性均具有显著影响。根部水淹条件下 高土壤养分处理提高了植株根生物量的积累, 该效 应在低高程植株中更为显著。高低高程植株表现出 不同的生长和繁殖策略, 高高程植株将更多的生物 量投入到营养生长, 而低高程植株则将更多的生物 量投入到生殖生长。研究结果表明该物种对根部水 淹条件下高土壤养分环境具有较好的适应性; 同时， 低高程植株可通过调整其生长和繁殖性状以提高其 对环境的适应性。

\section{参考文献}

Abbott JM, Stachowicz JJ (2016). The relative importance of trait vs. genetic differentiation for the outcome of interactions among plant genotypes. Ecology, 97, 84-94.

Ayi QL, Zeng B, Yang K, Lin F, Zhang XP, van Bodegom PM, Cornelissen JHC (2019). Similar growth performance but

www.plant-ecology.com 
contrasting biomass allocation of root-flooded terrestrial plant Alternanthera philoxeroides (Mart.) Griseb. in response to nutrient versus dissolved oxygen stress. Frontiers in Plant Science, 10, 111. DOI: 10.3389/fpls.2019.00111.

Barker HL, Holeski LM, Lindroth RL (2019). Independent and interactive effects of plant genotype and environment on plant traits and insect herbivore performance: a meta-analysis with Salicaceae. Functional Ecology, 33, 422-435.

Chen FQ, Guan SP, Ma YR, Xie ZQ, Lü K, Huang YW, Jia GM (2019a). Impact of regulated water level fluctuations on the sexual reproduction of remnant Myricaria laxiflora populations. Global Ecology and Conservation, 18, e00628. DOI: 10.1016/j.gecco.2019.e00628.

Chen FQ, Li Y, Qie GW, Xu WN (2008). The morphological responses and endurance of Polygonum hydropiper to flooding stress. Journal of Wuhan Botanical Research, 26, 142-146. [陈芳清, 李永, 郄光武, 许文年 (2008). 水苶 对水淹胁迫的耐受能力和形态学响应. 武汉植物学研 究, 26, 142-146.]

Chen FQ, Xie ZQ (2007). Reproductive allocation, seed dispersal and germination of Myricaria laxiflora, an endangered species in the Three Gorges Reservoir area. Plant Ecology, 191, 67-75.

Chen FQ, Xie ZQ (2011). Ecophysiological Response of Two Herbaceous Species to Flooding Implication for Ecological Restoration of Vegetation on Water-level-fluctuating Zone. International Conference on Electronics, Communications and Control (ICECC). Ningbo, China. 4260-4263.

Chen FQ, Zhang M, Wu Y, Huang YW (2020). Seed rain and seed bank of a draw-down zone and their similarities to vegetation under the regulated water-level fluctuation in Xiangxi River. Journal of Freshwater Ecology, 35, 57-71.

Chen XS, Deng ZM, Xie YH, Li F, Li X (2014). Differential growth and vegetative reproduction by two co-occurring emergent macrophytes along a water table gradient. Pakistan Journal of Botany, 46, 881-886.

Chen XS, Li YF, Cai YH, Xie YH, Deng ZM, Li F, Hou ZY (2019b). Differential strategies to tolerate flooding in Polygonum hydropiper plants originating from low- and high-elevation habitats. Frontiers in Plant Science, 9, 1970. DOI: 10.3389/fpls.2018.01970.

Cheng RM, Liu ZB, Xiao WF, Wang N, Wang XR, Shen YF (2017). Changes of soil chemical properties in typical hydro-fluctuation belt of Three Gorges Reservoir. Scientia Silvae Sinicae, 53(2), 19-25. [程瑞梅, 刘泽涁, 肖文发, 王娜, 王晓荣, 沈雅飞 (2017). 三峡库区典型消落带土 壤化学性质变化. 林业科学, 53(2), 19-25.]

Colmer TD, Voesenek LACJ (2009). Flooding tolerance: suites of plant traits in variable environments. Functional Plant Biology, 36, 665-681.

Du H, Zhang XP, Zeng B (2016). Dissolved oxygen in water affects the tolerance of two terrestrial plants, Alternanthera philoxeroides and Hemarthria altissima, to complete submergence. Acta Ecologica Sinica, 36, 7562-7569. [杜 珲, 张小萍, 曾波 (2016). 水体溶氧影响陆生植物喜旱 莲子草(Alternanthera philoxeroides)和牛鞭草(Hemarthria altissima)对完全水淹的耐受力. 生态学报, 36, 7562-7569.]

Fan DY, Xiong GM, Zhang AY, Liu X, Xie ZQ, Li ZJ (2015). Effect of water-lever regulation on species selection for ecological restoration practice in the water-level fluctuation zone of Three Gorges Reservoir. Chinese Journal of Plant Ecology, 39, 416-432. [樊大勇, 熊高明, 张爱英, 刘曦, 谢宗强, 李兆佳 (2015). 三峡库区水位 调度对消落带生态修复中物种笁选实践的影响. 植物 生态学报, 39, 416-432.]

Fan SF, Yu HH, Liu CH, Yu D, Han YQ, Wang LG (2015). The effects of complete submergence on the morphological and biomass allocation response of the invasive plant Alternanthera philoxeroides. Hydrobiologia, 746, 159-169.

Fan XL, Yang TH, Gao HY, Zhang ZS, Yang C, Liu MJ (2014). Effect of oxygen on the damage of photosynthetic apparatus in plant leaves induced by dark-submergence. Plant Physiology Journal, 50, 542-548. [樊杏利, 杨天慧, 高辉远, 张子山, 杨程, 刘美君 (2014). 氧气在黑暗-水 淹诱导植物叶片光合机构损伤中的作用. 植物生理学 报, 50, 542-548.]

Gu YB, Pan YW, Chen FY, Lou YJ, Tang ZH (2019). Effects of water level and nitrogen concentration on growth and biomass allocation of Scirpus nipponicus seedlings. Chinese Journal of Ecology, 38, 2302-2309. [古勇波, 潘 艳文, 陈方圆, 娄彦景, 唐占辉 (2019). 水位和氮浓度 对三江蔍草幼苗生长和生物量分配的影响. 生态学杂 志, 38, 2302-2309.]

Guo Y, Yang S, Shen YF, Xiao WF, Cheng RM (2019). Study on the natural distribution characteristics and community species diversity of existing plants in the Three Gorges Reservoir. Acta Ecologica Sinica, 39, 4255-4265. [郭燕, 杨邵, 沈雅飞, 肖文发, 程瑞梅 (2019). 三峡水库消落 带现存植物自然分布特征与群落物种多样性研究. 生 态学报, 39, 4255-4265.]

He RT, Yang K, Zeng B, Li R, Niu HG, Shi SH, Ayi QL, Su XL (2020). Distribution pattern of vegetation in water-level fluctuation zone of the Three Gorges Reservoir as affected by differential flooding regimes. Acta Ecologica Sinica, 40, 834-842. [何芯廷，杨康，曾波，李 瑞，牛汉刚，史邵华，阿依巧丽，苏晓磊 (2020). 三峡 水库消落区植被在差异性水淹环境中的分布格局. 生 态学报, 40, 834-842.]

Heydel F, Engels JG, Feigs JT, Vásquez E, Rudolph B, Rohwer JG, Jensen K (2017). Adaptation to tidal flooding and rapid genetic divergence between a narrow endemic grass species and its widespread congener lead to an early stage of ecological speciation. Perspectives in Plant Ecology, Evolution and Systematics, 27, 57-67. 
Hong M, Guo QS, Nie BH, Kang Y, Pei SX, Jin JQ, Wang XF (2011). Responses of Cynodon dactylon population in hydro-fluctuation belt of Three Gorges Reservoir area to flooding-drying habitat change. Chinese Journal of Applied Ecology, 22, 2829-2835. [洪明, 郭泉水, 聂必 红, 康义, 裴顺祥, 金江群, 王祥福 (2011). 三峡库区 消落带狗牙根种群对水陆生境变化的响应. 应用生态 学报, 22, 2829-2835.]

Hua JF, Han LW, Wang ZQ, Gu CS, Yin YL (2017). Morpho-anatomical and photosynthetic responses of Taxodium hybrid 'Zhongshanshan' 406 to prolonged flooding. Flora, 231, 29-37.

Lei B, Wang YC, You YF, Zhang S, Yang CH (2014). Diversity and structure of herbaceous plant community in typical water-level-fluctuation zone with different spacing elevations in Three Gorges Reservior. Journal of Lake Sciences, 26，600-606. [雷波，王业春，由永飞，张晟， 杨春华 (2014). 三峡水库不同间距高程消落带草本植 物群落物种多样性与结构特征. 湖泊科学, 26 , 600-606.]

Lenssen JPM, van Kleunen M, Fischer M, de Kroon H (2004). Local adaptation of the clonal plant Ranunculus reptans to flooding along a small-scale gradient. Journal of Ecology, 92, 696-706.

Li AR (1998). Flora of China: Volume 25th, 1st Fascicle. Science Press, Beijing. 27. [李安仁 (1998). 中国植物志: 第25卷第1分册. 科学出版社, 北京. 27.]

Li QH, Liu SP, Zhi CY, Li XF, Chen FF, Zeng QK (2013). Adaptation mechanism of three herbs in the water-levelfluctuation-zone of reservoir to complete submergence. Journal of Tropical and Subtropical Botany, 21, 459-465. [李秋华, 刘送平, 支崇远, 李小峰, 陈峰峰, 曾庆凯 (2013). 三种水库消落带草本植物对完全水淹的适应机 制研究. 热带亚热带植物学报, 21, 459-465.]

Li XX, Li CX, Song H, Yuan ZX (2019). Effects of flooding and planting density on the photosynthesis of Hemarthria compressa and Cynodon dactylon cottage seedlings. Acta Prataculturae Sinica, 28, 197-206. [李晓雪, 李昌晓, 宋 虹, 袁中勋 (2019). 水淹和密度配置对牛鞭草与狗牙根 扞插苗光合作用的影响. 草业学报, 28, 197-206.]

Li YF, Chen XS, Xiang WH, Xie YH (2016). Effects of water levels on the growth and reproductive characteristics of Carex brevicuspis growing on sites with different elevations. Acta Ecologica Sinica, 36, 1959-1966. [李亚 芳, 陈心胜, 项文化, 谢永宏 (2016). 不同高程短尖苔 草对水位变化的生长及繁殖响应. 生态学报, 36, 1959-1966.]

Liu WW, Wang J, Wang Y, Yang F (2012). The differences of plant community diversity among the different altitudes in the water-level-fluctuating zone of the Three Gorges Reservoir. Acta Ecologica Sinica, 32, 5454-5466. [刘维 暐, 王杰, 王勇, 杨帆 (2012). 三峡水库消落区不同海
拔高度的植物群落多样性差异. 生态学报, 32, 5454-5466.]

Liu ZB, Cheng RM, Xiao WF, Wang RL, Feng XH, Wang XR (2013). Effect of waterlogging on photosynthetic and physioecological characteristics of plants. World Forestry Research, 26, 33-38. [刘泽涁, 程瑞梅, 肖文发, 王瑞丽, 封晓辉, 王晓荣 (2013). 水淹胁迫对植物光合生理生态 的影响. 世界林业研究, 26, 33-38.]

Luo MJ, Zhang SG, Cui LJ, Tan FL, Huang YR (2012). Response of growth and biomass allocation of Aegiceras corniculatum to waterlogging stress. Journal of Zhejiang Forestry Science and Technology, 32, 15-19. [罗美娟, 张 守攻, 崔丽娟, 谭芳林, 黄雍容 (2012). 桐花树幼苗生 长与生物量分配对淹水胁迫的响应. 浙江林业科技, 32 , 15-19.]

Mony C, Mercier E, Bonis A, Bouzillé JB (2010). Reproductive strategies may explain plant tolerance to inundation: a mesocosm experiment using six marsh species. Aquatic Botany, 92, 99-104.

Nam JM, Kim JH, Kim JG (2017). Effects of light intensity and plant density on growth and reproduction of the amphicarpic annual Persicaria thunbergii. Aquatic Botany, $142,119-122$.

Pan Y, Xie YH, Deng ZM, Tang Y, Pan DD (2014). High water level impedes the adaptation of Polygonum hydropiper to deep burial: responses of biomass allocation and root morphology. Scientific Reports, 4, 5612. DOI: 10.1038/ srep05612.

Park H, Kim JG (2020). Temporal and spatial variations of vegetation in a riparian zone of South Korea. Journal of Ecology and Environment, 44, 9. DOI: 10.1186/s41610020-00152-z.

Phukan UJ, Mishra S, Shukla RK (2016). Waterlogging and submergence stress: affects and acclimation. Critical Reviews in Biotechnology, 36, 956-966.

Qin HW, Gao F, Liu ZX, Li HL, Zheng LD, Su HY, Meng JM (2017). Effects of light intensity on the growth and physiology of Cynodon dactylon seedlings under water submerged environment. Acta Agrestia Sinica, 25, 675-679. [秦洪文, 高芳, 刘正学, 李洪林, 郑丽丹, 苏 华英, 孟佳媚 (2017). 水淹环境下光强对狗牙根幼苗生 长及生理的影响. 草地学报, 25, 675-679.]

Schaff SD, Pezeshki SR, Shields FD (2003). Effects of soil conditions on survival and growth of black willow cuttings. Environmental Management, 31, 748-763.

Soininen EM, Bråthen KA, Jusdado JGH, Reidinger S, Hartley SE (2013). More than herbivory: levels of silica-based defences in grasses vary with plant species, genotype and location. Oikos, 122, 30-41.

Striker GG, Manzur ME, Grimoldi AA (2011). Increasing defoliation frequency constrains regrowth of the forage legume Lotus tenuis under flooding. The role of crown

www.plant-ecology.com 
reserves. Plant and Soil, 343, 261-272.

Su XL, Nilsson C, Pilotto F, Liu SP, Shi SH, Zeng B (2017). Soil erosion and deposition in the new shorelines of the Three Gorges Reservoir. Science of the Total Environment, 599-600, 1485-1492.

Su XL, Zeng B, Huang WJ, Xu SJ, Lei ST (2012). Effects of the Three Gorges Dam on preupland and preriparian drawdown zones vegetation in the upper watershed of the Yangtze River, P. R. China. Ecological Engineering, 44, 123-127.

Su XL, Zeng B, Lin F, Qiao P, Ayi QL, Huang WJ (2016). How does long-term complete submergence influence sex ratio and resource allocation of a dioecious shrub, Salix variegata Franch.? Ecological Engineering, 87, 218-223.

Su XL, Zeng B, Qiao P, Ayi QL, Huang WJ (2010). The effects of winter water submergence on flowering phenology and reproductive allocation of Salix variegata Franch. in Three Gorges reservoir region. Acta Ecologica Sinica, 30, 2585-2592. [苏晓磊, 曾波, 乔普, 阿依巧丽, 黄文军 (2010). 冬季水淹对秋华柳的开花物候及繁殖分配的影 响. 生态学报, 30, 2585-2592.]

Tao M, Bao DC, Jiang MX (2011). Effects of submergence on seed germination of nine annual plant species in the Three Gorges Reservoir region and their implication to vegetation restoration. Acta Ecologica Sinica, 31, 906-913. [陶敏, 鲍大川, 江明喜 (2011). 三峡库区9种 植物种子萌发特性及其在植被恢复中的意义. 生态学 报, 31, 906-913.]

Villellas J, García MB (2018). Life-history trade-offs vary with resource availability across the geographic range of a widespread plant. Plant Biology, 20, 483-489.

Voesenek LACJ, Colmer TD, Pierik R, Millenaar FF, Peeters AJM (2006). How plants cope with complete submergence. New Phytologist, 170, 213-226.

Wang C, Fang F, Yuan ZY, Zhang R, Zhang W, Guo JS (2020). Spatial variations of soil phosphorus forms and the risks of phosphorus release in the water-level fluctuation zone in a tributary of the Three Gorges Reservoir. Science of the Total Environment, 699, 134124. DOI: 10.1016/j.scitotenv. 2019.134124.

Wang P, Zhang Q, Xu YS, Yu FH (2016). Effects of water level fluctuation on the growth of submerged macrophyte communities. Flora, 223, 83-89.

Wang YC, Lei B, Zhang S (2012). Differences in vegetation and soil characteristics at different water-level altitudes in the drawdown areas of Three Gorges Reservoir area. Journal of Lake Sciences, 24, 206-212. [王业春, 雷波, 张晟 (2012). 三峡库区消落带不同水位高程植被和土 壤特征差异. 湖泊科学, 24, 206-212.]

Wang YJ, Chen FQ, Zhang M, Chen SH (2016a). Response of soil nutrient levels and spatial distribution to water-level fluctuation on the shanmu riverbanks in the Three Gorges
Reservoir area. Journal of Hydroecology, 37, 56-61. [王娅 儆, 陈芳清, 张永, 陈韶华 (2016a). 三峡库区水位消涨 对杉木溪消落带土壤性质的影响. 水生态学杂志, 37 , 56-61.]

Wang YJ, Chen FQ, Zhang M, Wu Y, Chen SH (2016b). Characteristics of soil nutrient and spatial distribution on riparian zone restored by different vegetation restoration methods at Wanzhou section in the Three Gorges Reservoir area, China. Journal of Agricultural Resources and Environment, 33, 127-133. [王娅儆, 陈芳清, 张永, 吴阳，陈韶华 (2016b). 不同植被恢复模式下三峡库区 万州段消落带土壤养分及其空间分布特征. 农业资源 与环境学报, 33, 127-133.]

Wei GW, Sun XS, Chen YH, Luo FL, Yu FH (2020). Growth and reproductive responses of Polygonum hydropiper populations to elevational difference associated with flooding. Global Ecology and Conservation, 23, e01156. DOI: 10.1016/j.gecco.2020.e01156.

Wu JG, Huang JH, Han XG, Gao XM, He FL, Jiang MX, Jiang ZG, Primack RB, Shen ZH (2004). The Three Gorges Dam: an ecological perspective. Frontiers in Ecology and the Environment, 2, 241-248.

Xing W, Yin M, Lü Q, Hu Y, Liu CP, Zhang JJ (2014). Oxygen solubility, diffusion coefficient, and solution viscosity// Xing W, Yin GP, Zhang JJ. Rotating Electrode Methods and Oxygen Reduction Electrocatalysts. Elsevier, Amsterdam. 1-31.

Xu JP, Zhang XP, Zeng B, Yuan SH, Liu JH, Liu MZ (2014). Effects of light and dissolved oxygen on the phenotypic plasticity of Alternanthera philoxeroides in submergence conditions. Acta Ecologica Sinica, 34, 258-268. [许建平, 张小萍, 曾波, 袁慎鸿, 刘建辉, 刘明智 (2014). 完全 水淹环境中光照和溶氧对喜旱莲子草表型可塑性的影 响. 生态学报, 34, 258-268.]

Xu JY, Chen HM, Wang XL (2016). A review on water depth effect on the growth and reproduction of plants in the wetlands. Wetland Science, 14, 725-732. [徐金英, 陈海 梅, 王晓龙 (2016). 水深对湿地植物生长和繁殖影响研 究进展. 湿地科学, 14, 725-732.]

Yan H, Liu RQ, Liu ZN, Wang X, Luo WB, Sheng LX (2015). Growth and physiological responses to water depths in Carex schmidtii meinsh. PLOS ONE, 10, e0128176. DOI: 10.1371/journal.pone.0128176.

Ye C, Chen CR, Butler OM, Rashti MR, Esfandbod M, Du M, Zhang QF (2019). Spatial and temporal dynamics of nutrients in riparian soils after nine years of operation of the Three Gorges Reservoir, China. Science of the Total Environment, 664, 841-850.

Ye XQ, Zeng B, Meng JL, Wu M, Zhang XP (2018). Responses in shoot elongation, carbohydrate utilization and growth recovery of an invasive species to submergence at different water temperatures. Scientific 
Reports, 8, 306. DOI: 10.1038/s41598-017-18735-7.

You YF, Yang CH, Lei B, Zhang S, Wang YC, Liu JH (2017). Effect of water level regulation on vegetation characteristics in the water-level-fluctuation zone of the Three Gorges Reservoir. Chinese Journal of Applied and Environmental Biology, 23, 1103-1109. [由永飞, 杨春华, 雷波, 张晟, 王业春, 刘建辉 (2017). 水位调节对三峡 水库消落带植被群落特征的影响. 应用与环境生物学 报, 23, 1103-1109.]

Yuan SH, Zeng B, Su XL, Xu JP (2014). Effect of water-level fluctuation discrepancy on the composition of different annuals in Three Gorges Reservoir drawdown zone. Acta Ecologica Sinica，34，6481-6488. [袁慎鸿，曾波，苏晓 磊，许建平 (2014). 水位节律差异对三峡水库消落区不 同物候类型 1 年生植物物种构成的影响. 生态学报, 34 , 6481-6488.]

Zhang AY, Xiong GM, Fan DY, Yang D, Xie ZQ (2018). Effects of Three Gorges Dam on riparian vascular plants of the main stream of Yangtze River. Resources and Environment in the Yangtze Basin, 27, 145-156. [张爱英, 熊高明, 㚞大勇, 杨丹, 谢宗强 (2018). 三峡水库蓄水 对长江干流河岸植物组成的影响. 长江流域资源与环 境, 27, 145-156.]

Zhang Q, Peters JL, Visser EJW, de Kroon H, Huber H (2016). Hydrologically contrasting environments induce genetic but not phenotypic differentiation in Solanum dulcamara. Journal of Ecology, 104, 1649-1661.

Zhang Y, Cai JG, Sun OW, Shi JJ (2019). Research on photosynthetic responses mechanisms of Hydrangea macrophylla under waterlogging stress. Journal of Nuclear
Agricultural Sciences, 33, 808-815. [章毅, 蔡建国, 孙欧 文, 施健健 (2019). 水淹胁迫下绣球光合响应机制的研 究. 核农学报, 33, 808-815.]

Zhang ZY, Cheng YC, Cheng L, Wan CY, Li JB (2016). Characteristics of vegetation and soil in the water level fluctuation zone of the Wanzhou region of Three Gorges Reservoir. Journal of Hydroecology, 37, 24-33. [张志永, 程郁春, 程丽, 万成炎, 李金波 (2016). 三峡库区万州 段消落带植被及土壤理化特征分析. 水生态学杂志, 37 , 24-33.]

Zhao Q, Chen JB (2018). Study and practice on ecological restoration strategy of the hydro-fluctuation belt in the Three Gorges Reservoir area. Journal of Anhui Agricultural Sciences, 46, 5-7. [赵琴, 陈教斌 (2018). 三 峡库区消落带生态修复策略研究与实践. 安徽农业科 学, 46, 5-7.]

Zhou WG, Chen F, Meng YJ, Chandrasekaran U, Luo XF, Yang WY, Shu K (2020). Plant waterlogging/flooding stress responses: from seed germination to maturation. Plant Physiology and Biochemistry, 148, 228-236.

Zhu Q, Zhang ZY, Hu HQ, Wan CY, Hu L, Liu H (2014). Soil property changes under drain-flooding condition in Xiaojiang water-level-fluctuating belt of the Three-GorgeReservoir region. Soils, 46, 927-932. [朱强, 张志永, 胡 红青, 万成炎, 胡莲, 刘晖 (2014). 淹没-出露条件下三 峡水库小江消落带土壤性质变化研究.土壤, 46, 927-932.]

责任编委: 曾 波 责任编辑: 李 敏

附录I 三峡水库2009-2018年蓄水期间高流量引起的水位上涨速率 $\left(\mathbf{m} \cdot \mathbf{d}^{-\mathbf{1}}\right)$ Supplement I Water-level rise rate caused by the high flow of the Three Gorges Reservoir from 2009 to $2018\left(\mathrm{~m}^{\circ} \mathrm{d}^{-1}\right)$

\begin{tabular}{llllllllll}
\hline 2009 & 2010 & 2011 & 2012 & 2013 & 2014 & 2015 & 2016 & 2017 & 2018 \\
\hline 1.00 & 2.65 & 2.46 & 2.34 & 1.32 & 1.31 & 1.54 & 1.51 & 1.91 & 1.23 \\
1.38 & 3.04 & 2.49 & 2.46 & 1.50 & 1.34 & 1.55 & 1.31 & 1.65 & 1.06 \\
1.91 & 4.00 & 2.70 & 3.15 & 1.62 & 3.80 & 2.47 & 1.25 & 1.48 & 1.02
\end{tabular}

数据为每年水库蓄水期间水位上涨最快 3 天的数据。水位上涨速率根据2009-2018年全国水雨情信息网(http://xxfb.mwr.cn/sq djdh.html)每日水位数据计 算得出。

Data shown are the water-level rise rate of the fastest three days during the impoundment of the reservoir each year. The water level rise rate was calculated based on the daily water level data of the National Water and Rain Information Network (http://xxfb.mwr.cn/sq_djdh.html) from 2009 to 2018. 